\title{
Towards innovative, successful and cost-efficient use of beam time
}

This double issue presents some of the work accomplished by two Joint Research Activities (JRAs) of the project World class Science and Innovation with Neutrons in Europe 2020 (SINE2020) financed by the European Commission under the 8th Framework Programme. Within these two JRAs entitled E-tools for Instrumentation and Sample Environment, efforts have been made to explore the full potential of the large scale facilities and enable challenging experiments required by the neutron and muon user communities.

In the following pages, we present new McStas components based on Monte-Carlo algorithms to optimise the signal to background ratio, a decisive factor for the success of many experiments. The first McStas bundle allows evaluating one of the major contributions to dose rate around neutron guides. Combined with the next article presenting the investigation of the neutron activation properties of the PE-B4C-concrete recently developed for the European Spallation Source (ESS), this will help facilities to optimise the siting of guides and instruments.

The second presented McStas bundle calculates neutron pathways through materials surrounding samples which are generally the most difficult to screen. For the first time, textured materials are considered, taking elastic and inelastic scattering into account, including multiple scattering. The article is followed by a review of different materials for building novel pressure cells for inelastic scattering whose measurements have allowed to determine the effective phonon generalised density of states of these materials, a prerequisite for performing simulations.

The next two articles focus on pressure devices. The first one presents the developments of gasket-anvil assemblies for the Paris-Edinburgh cell with 30\% improved pressure performance giving a similar signal-to-background ratio. It also describes an improved Bridgman seal package which makes the low-temperature operation of the PE press significantly more reliable. The second article describes a new cell for the investigation of hydrogen storage materials at pressures up to 700 bar and temperatures up to $500^{\circ} \mathrm{C}$.

The following articles present a simple but very efficient technique for reducing the cool-down times of blue series furnaces by a factor 4 to 5, a comprehensive suite of sample environment equipment for efficient running of muon chemistry experiments over a wide range of conditions, and a portable single-sided NMR setup adapted to a small angle neutron scattering instrument for retrieving both structural and dynamical informations from a single experiment.

This issue ends with an article demonstrating that rectangular beam cross-sections with a height over width ratio which mimic the ESS "pancake" moderator beams will lead to an improved homogeneity of the magnetic field integrals of spin echo instruments and an introduction to SECoP, the international Sample Environment Communication Protocol developed on close collaboration with the International Society for Sample Environment.

Enjoy the reading!

E. Lelièvre-Berna Institut Laue-Langevin

P. Willendrup Technical University of Denmark European Spallation Source 